International Journal of Geometric Methods in Modern Physics

(C) World Scientific Publishing Company

\title{
Fourier transform from momentum space to twistor space
}

\author{
Jun-ichi Note \\ Research Institute of Science 83 Technology, College of Science and Technology, \\ Nihon University, Tokyo 101-8308, Japan \\ note.junichi20@nihon-u.ac.jp
}

\begin{abstract}
Several methods use the Fourier transform from momentum space to twistor space to analyze scattering amplitudes in Yang-Mills theory. However, the transform has not been defined as a concrete complex integral when the twistor space is a three-dimensional complex projective space. To the best of our knowledge, this is the first study to define it as well as its inverse in terms of a concrete complex integral. In addition, our study is the first to show that the Fourier transform is an isomorphism from the zeroth Cech cohomology group to the first one. Moreover, the well-known twistor operator representations in twistor theory literature are shown to be valid for the Fourier transform and its inverse transform. Finally, we identify functions over which the application of the operators is closed.
\end{abstract}

Keywords: Fourier transform; Twistor space; Čech cohomology group.

\section{Introduction}

The introduction of twistor string theory by Witten in 2004 [1] triggered the development of new methods for analyzing scattering amplitudes in Yang-Mills theory and gravity theory [2. In twistor string theory, the tree-level maximally helicity violating $(\mathrm{MHV})$ amplitudes of four-dimensional $\mathcal{N}=4$ super Yang-Mills (SYM) theory are analyzed by applying a Fourier transform from momentum space to twistor space. This transform is sometimes referred to as a half-Fourier transform [3] because it is a transform with respect to the spinor variable $\tilde{\pi}_{\alpha}$ alone in the light-like momentum vector $p_{\alpha \dot{\alpha}}=\tilde{\pi}_{\alpha} \pi_{\dot{\alpha}}$, where $\alpha=0,1$ and $\dot{\alpha}=\dot{0}, \dot{1}$. It has been shown that a gluon MHV amplitude in twistor space is supported on an algebraic curve of degree one. This amplitude is deduced from the string model whose target space is the twistor space. In general, it is conjectured that an $l$-loop amplitude with $q$ gluons of negative helicity in twistor space is supported on an algebraic curve of degree $d=q-1+l$. Another example that demonstrates the utility of the Fourier transform is the translation of the Britto-Cachazo-Feng-Witten (BCFW) recursion relations 45 , concerning scattering amplitudes in momentum space. This shows that scattering amplitudes in twistor space have the same recursion relations 617. In addition, the Fourier transform is also used in the representation of the $n$-particle (next-to) ${ }^{k}$ maximally helicity violating ( $\left.\mathrm{N}^{k} \mathrm{MHV}\right)$ amplitude , i.e., the amplitude with $k+2$ negative helicity gluons and $n-k-2$ positive helicity gluons, 
in planar $\mathcal{N}=4 \mathrm{SYM}$ theory as an integral on a Grassmann manifold with the aid of twistors [8]. This makes the symmetries of the $\mathrm{N}^{k} \mathrm{MHV}$ amplitude, i.e., the cyclic symmetry of the external particles, the superconformal symmetry, and the Yangian symmetry, manifest. Furthermore, relativistic particles of various helicities can be analyzed similarly in terms of twistor variables with the aid of the Fourier transform 10.

When the spacetime metric signature is $(2,2)$, i.e., the ultra-hyperbolic spacetime, the spinors $\pi_{\dot{\alpha}}$ and $\tilde{\pi}_{\alpha}$ of the light-like momentum vector are real, and the corresponding twistor space is the three-dimensional real projective space $\mathbb{R P}^{3}[1$. Thus, the Fourier transform from momentum space to twistor space is the wellknown one from the standard analysis. However, when the spacetime metric signature is $(1,3)$, i.e., the Minkowski space $\mathbb{M}$, the spinor $\pi_{\dot{\alpha}}$ is complex and the spinor $\tilde{\pi}_{\alpha}$ is its complex conjugate, i.e., $\tilde{\pi}_{\alpha}=\bar{\pi}_{\alpha}$, and the corresponding twistor space is the three-dimensional complex projective space $\mathbb{C P}^{3}[11$. In this case, the Fourier transform is a complex integral with respect to the spinor variable $\bar{\pi}_{\alpha}$. Hence, the definition is different from that in the real-number case. Thus far, this Fourier transform has not been defined as a concrete complex integral [810. Therefore, the concrete integration method, the functions to which the integral can be applied, and the explicit results of the integral are all unknown. Investigating these issues could facilitate studies of the mathematical structures of the scattering amplitudes in $\mathcal{N}=4 \mathrm{SYM}$ theory as well as those of relativistic particles, which remain unknown.

This paper proposes a new definition of the Fourier transform as a concrete complex integral of functions in momentum space in the following way. First, we assume that the momentum-space functions $f\left(\bar{\pi}_{\alpha}, \pi_{\dot{\alpha}}\right)$ can be expanded in terms of positive powers $\bar{\pi}_{\alpha},\left(\bar{\pi}_{0}\right)^{a}\left(\bar{\pi}_{1}\right)^{b}(a, b=0,1,2, \cdots)$. Here, we denote the momentum-space coordinates by $\left(\bar{\pi}_{\alpha}, \pi_{\dot{\alpha}}\right)$. Then, we propose the Fourier transform of $f\left(\bar{\pi}_{\alpha}, \pi_{\dot{\alpha}}\right)$ as a concrete complex integral with respect to the variables $\bar{\pi}_{\alpha}$ such that it transforms $f\left(\bar{\pi}_{\alpha}, \pi_{\dot{\alpha}}\right)$ to a function of twistor-space coordinates $\left(\omega^{\alpha}, \pi_{\dot{\alpha}}\right)$, where we define the spinor $\omega^{\alpha}$ by contracting $\pi_{\dot{\alpha}}$ with the coordinates $\left(x^{\alpha \dot{\alpha}}\right)$ of $\mathbb{M}$, i.e., $\omega^{\alpha}:=i x^{\alpha \dot{\alpha}} \pi_{\dot{\alpha}}$. Here, we determine the integral contour such that $\left(\bar{\pi}_{0}\right)^{0}\left(\bar{\pi}_{1}\right)^{0}=1$ is transformed to a complex function that represents the delta function of Sato's hyperfunction. In this definition, the Fourier transform of $f\left(\bar{\pi}_{\alpha}, \pi_{\dot{\alpha}}\right)$ is the twistor-space function $\tilde{f}\left(\omega^{\alpha}, \pi_{\dot{\alpha}}\right)$ expanded in terms of negative powers $\omega^{\alpha},\left(\omega^{0}\right)^{-a-1}\left(\omega^{1}\right)^{-b-1}$. Next, we propose an inverse Fourier transform of twistor-space functions $\tilde{f}\left(\omega^{\alpha}, \pi_{\dot{\alpha}}\right)$ to momentum space. We define it as a concrete complex integral that transforms a complex function on twistor space that represents the delta function of Sato's hyperfunction to 1. In this definition, the momentum-space function $f\left(\bar{\pi}_{\alpha}, \pi_{\dot{\alpha}}\right)$ is obtained from the inverse Fourier transform of the function $\tilde{f}\left(\omega^{\alpha}, \pi_{\dot{\alpha}}\right)$, which is the Fourier transform of $f\left(\bar{\pi}_{\alpha}, \pi_{\dot{\alpha}}\right)$. Furthermore, the twistor-space function $\tilde{f}\left(\omega^{\alpha}, \pi_{\dot{\alpha}}\right)$ is obtained from the Fourier transform of the function $f\left(\bar{\pi}_{\alpha}, \pi_{\dot{\alpha}}\right)$, which is the inverse Fourier transform of $\tilde{f}\left(\omega^{\alpha}, \pi_{\dot{\alpha}}\right)$. In other words, this inverse Fourier transform is precisely the inverse map of the Fourier transform. 
From the perspective of the Cech cohomology group, the power functions $\left(\bar{\pi}_{0}\right)^{a}\left(\bar{\pi}_{1}\right)^{b}(a+b=n, n=0,1,2, \cdots)$ to which the Fourier transform is applied form a basis for the zeroth Cech cohomology group on $\mathbb{C P}^{1}$ with coefficients in the sheaf $\mathcal{O}(n)$ consisting of homogeneous functions of degree $n$. Furthermore, the power functions $\left(\omega^{0}\right)^{-a-1}\left(\omega^{1}\right)^{-b-1}$, which are obtained from the Fourier transform of $\left(\bar{\pi}_{0}\right)^{a}\left(\bar{\pi}_{1}\right)^{b}$, form a basis for the first Cech cohomology group on $\mathbb{C P}^{1}$ with coefficients in the sheaf $\mathcal{O}(-n-2)$ consisting of homogeneous functions of degree $-n-2$. Accordingly, this study is the first to show that the Fourier transform is an isomorphism from the zeroth Čech cohomology group to the first one, and the momentum-space functions to which the Fourier transform is applied are direct sums of the tensor product of the two zeroth Čech cohomology groups. Furthermore, the twistor-space functions obtained from the Fourier transform are shown to be direct sums of the tensor product of the zeroth and first Cech cohomology groups.

In the canonical quantization procedure in twistor theory, the variables $\omega^{\alpha}$ and $\bar{\pi}_{\alpha}$ are replaced by their corresponding operators 12 13|14. As in standard quantum mechanics, one of these operator types is represented as a multiplication operator, and the other is represented as a partial differential operator. We show that such operator representations are also valid for the Fourier transform and its inverse. Finally, we prove that the application of these operators is closed in the momentumspace functions and twistor-space functions, respectively.

This paper is organized as follows. Sec. 2 proposes a new definition of the Fourier transform from momentum space to twistor space as well as its inverse. Sec. 3 describes the functions to which the (inverse) Fourier transform is applied in terms of Cech cohomology groups. Sec. 4] shows that the well-known operator representations in the twistor theory literature are also valid for the (inverse) Fourier transform. Finally, Sec. [5] summarizes our study.

\section{New definition of the Fourier transform}

\subsection{Brief review of the delta function of two variables}

The delta function of two variables can be defined as a product of single-variable delta functions [15:

$$
\begin{aligned}
\delta\left(x_{1}, x_{2}\right) & :=\delta\left(x_{1}\right) \delta\left(x_{2}\right) \\
& =\prod_{j=1}^{2} \frac{1}{-2 \pi i}\left(\frac{1}{x_{j}+i 0}-\frac{1}{x_{j}-i 0}\right) \\
& =\frac{1}{(-2 \pi i)^{2}} \sum_{\sigma} \frac{\operatorname{sgn} \sigma}{\left(x_{1}+i \sigma_{1} 0\right)\left(x_{2}+i \sigma_{2} 0\right)},
\end{aligned}
$$

where $\sigma=\left(\sigma_{1}, \sigma_{2}\right), \sigma_{1}, \sigma_{2}= \pm 1$, and $\operatorname{sgn} \sigma=\sigma_{1} \sigma_{2}$. By defining the $\sigma$ quadrant as

$$
\Gamma_{\sigma}=\left\{\left(\eta_{1}, \eta_{2}\right) \in \mathbb{R}^{2} \mid \sigma_{1} \eta_{1}>0, \sigma_{2} \eta_{2}>0\right\},
$$


Jun-ichi Note

each term in Eq. (1) can be interpreted as the boundary value of the two-variable complex function

$$
\frac{1}{(-2 \pi i)^{2}} \frac{\operatorname{sgn} \sigma}{z_{1} z_{2}}
$$

at $\mathbb{R}^{2}+i \Gamma_{(1,1)}, \mathbb{R}^{2}+i \Gamma_{(1,-1)}, \mathbb{R}^{2}+i \Gamma_{(-1,1)}$, and $\mathbb{R}^{2}+i \Gamma_{(-1,-1)}$.

In sections 2.2 and 2.3 , we propose a new definition of the Fourier transform and its inverse transform by use of Eq. (3).

\subsection{Fourier transform}

To derive a new definition of the Fourier transform as a concrete complex integral, we focus on momentum-space functions, which have the power series expansion

$$
f\left(\bar{\pi}_{\alpha}, \pi_{\dot{\alpha}}\right)=\sum_{a, b=0}^{\infty} C_{a b}\left(\pi_{\dot{0}}, \pi_{\dot{1}}\right)\left(\bar{\pi}_{0}\right)^{a}\left(\bar{\pi}_{1}\right)^{b}
$$

Here, $C_{a b}\left(\pi_{\dot{0}}, \pi_{\dot{1}}\right)$ denotes a holomorphic function of the spinor $\pi_{\dot{\alpha}}$. To transform such functions $f\left(\bar{\pi}_{\alpha}, \pi_{\dot{\alpha}}\right)$ to twistor space, we propose the Fourier transform from the power series terms $\left(\bar{\pi}_{0}\right)^{a}\left(\bar{\pi}_{1}\right)^{b}(a, b=0,1,2, \cdots)$ to functions of $\omega^{\alpha}$.

Definition 1. First, we propose the Fourier transform of $\left(\bar{\pi}_{0}\right)^{0}\left(\bar{\pi}_{1}\right)^{0}=1$ as follows:

$$
\begin{aligned}
\mathcal{F}[1] & :=\frac{1}{(-2 \pi i)^{2}} \int_{-i \Gamma_{\sigma}} e^{-\omega^{0} \bar{\pi}_{0}-\omega^{1} \bar{\pi}_{1}} d \bar{\pi}_{0} d \bar{\pi}_{1} \\
& =\frac{1}{(-2 \pi i)^{2}} \frac{s g n \sigma}{\omega^{0} \omega^{1}}, \quad\left(\omega^{0}, \omega^{1}\right) \in \mathbb{R}^{2}+i \Gamma_{\sigma} .
\end{aligned}
$$

This is a complex function similar to Eq. (3), which represents the delta function.

When we take $\sigma=(1,-1)$, the integral in Eq. (5) represents an improper integral from $-\infty$ to 0 on the imaginary axis for the variable $\bar{\pi}_{0}$ and from 0 to $\infty$ on the imaginary axis for the variable $\bar{\pi}_{1}$ :

$$
\begin{aligned}
& \frac{1}{(-2 \pi i)^{2}} \int_{-i \Gamma_{(1,-1)}} e^{-\omega^{0} \bar{\pi}_{0}-\omega^{1} \bar{\pi}_{1}} d \bar{\pi}_{0} d \bar{\pi}_{1} \\
& =\frac{1}{(-2 \pi i)^{2}} \int_{-\infty}^{0} e^{-\omega^{0}\left(i \operatorname{Im} \bar{\pi}_{0}\right)} d\left(i \operatorname{Im} \bar{\pi}_{0}\right) \int_{0}^{\infty} e^{-\omega^{1}\left(i \operatorname{Im} \bar{\pi}_{1}\right)} d\left(i \operatorname{Im} \bar{\pi}_{1}\right) \\
& =\frac{1}{(-2 \pi i)^{2}} \frac{-1}{\omega^{0} \omega^{1}}
\end{aligned}
$$

This is uniformly convergent on $\left(\omega^{0}, \omega^{1}\right) \in \mathbb{R}^{2}+i \Gamma_{(1,-1)}$. For all other $\sigma$, the meaning of the integral sign in Eq. (5) is the same, and the integral is uniformly convergent 
on $\left(\omega^{0}, \omega^{1}\right) \in \mathbb{R}^{2}+i \Gamma_{\sigma}$.

Theorem 2. The Fourier transform of the momentum-space function $f\left(\bar{\pi}_{\alpha}, \pi_{\dot{\alpha}}\right)$ is

$$
\begin{aligned}
& \mathcal{F}\left[f\left(\bar{\pi}_{\alpha}, \pi_{\dot{\alpha}}\right)\right] \\
& :=\frac{1}{(-2 \pi i)^{2}} \int_{-i \Gamma_{\sigma}} f\left(\bar{\pi}_{\alpha}, \pi_{\dot{\alpha}}\right) e^{-\omega^{0} \bar{\pi}_{0}-\omega^{1} \bar{\pi}_{1}} d \bar{\pi}_{0} d \bar{\pi}_{1} \\
& =\sum_{a, b=0}^{\infty} C_{a b}\left(\pi_{\dot{0}}, \pi_{\dot{1}}\right) \frac{s g n \sigma}{(-2 \pi i)^{2}} \frac{\Gamma(a+1) \Gamma(b+1)}{\left(\omega^{0}\right)^{a+1}\left(\omega^{1}\right)^{b+1}}, \quad\left(\omega^{0}, \omega^{1}\right) \in \mathbb{R}^{2}+i \Gamma_{\sigma} .
\end{aligned}
$$

By using the analytic continuation method, we can extend the $\left\{\left(\omega^{0}, \omega^{1}\right)\right\}$ region from $\mathbb{R}^{2}+i \Gamma_{\sigma}$ to the direct product of $\mathbb{C}^{*}=\left\{\omega^{0} \in \mathbb{C} \mid \omega^{0} \neq 0\right\}$ and $\mathbb{C}^{*}=\left\{\omega^{1} \in\right.$ $\left.\mathbb{C} \mid \omega^{1} \neq 0\right\}$. Hereafter, we consider the $\left\{\left(\omega^{0}, \omega^{1}\right)\right\}$ region in $E q$. (7) as $\mathbb{C}^{*} \times \mathbb{C}^{*}$.

Proof. From Eq. (5), the Fourier transform of the power series term $\left(\bar{\pi}_{0}\right)^{a}\left(\bar{\pi}_{1}\right)^{b}$ $(a, b=0,1,2, \cdots)$ is

$$
\begin{aligned}
\mathcal{F}\left[\left(\bar{\pi}_{0}\right)^{a}\left(\bar{\pi}_{1}\right)^{b}\right] & :=\frac{1}{(-2 \pi i)^{2}} \int_{-i \Gamma_{\sigma}}\left(\bar{\pi}_{0}\right)^{a}\left(\bar{\pi}_{1}\right)^{b} e^{-\omega^{0} \bar{\pi}_{0}-\omega^{1} \bar{\pi}_{1}} d \bar{\pi}_{0} d \bar{\pi}_{1} \\
& =\frac{\operatorname{sgn} \sigma}{(-2 \pi i)^{2}} \frac{\Gamma(a+1) \Gamma(b+1)}{\left(\omega^{0}\right)^{a+1}\left(\omega^{1}\right)^{b+1}}, \quad\left(\omega^{0}, \omega^{1}\right) \in \mathbb{R}^{2}+i \Gamma_{\sigma} .
\end{aligned}
$$

The integral in Eq. (8) can be calculated in the same way as that in Eq. (5). Here, the integration formula

$$
\int_{0}^{\infty} x^{z-1} e^{-w x} d x=\frac{\Gamma(z)}{w^{z}}, \quad \operatorname{Re} w>0, \quad \operatorname{Re} z>0
$$

has been used. Thus, from Eqs. (44) and (8), Eq. (77) is obtained.

\subsection{Inverse Fourier transform}

Now, we propose the inverse Fourier transform from twistor space to momentum space. Here, we focus on twistor-space functions $\tilde{f}\left(\omega^{\alpha}, \pi_{\dot{\alpha}}\right)$ that can be expanded in terms of the power series terms $\left(\omega^{0}\right)^{-a-1}\left(\omega^{1}\right)^{-b-1}(a, b=0,1,2, \cdots)$, similar to Eq. (7):

$$
\tilde{f}\left(\omega^{\alpha}, \pi_{\dot{\alpha}}\right)=\sum_{a, b=0}^{\infty} \frac{D_{a b}\left(\pi_{\dot{0}}, \pi_{\dot{\mathrm{i}}}\right)}{\left(\omega^{0}\right)^{a+1}\left(\omega^{1}\right)^{b+1}} .
$$

Here, $D_{a b}\left(\pi_{\dot{0}}, \pi_{\dot{1}}\right)$ is a holomorphic function of $\pi_{\dot{0}}$ and $\pi_{\dot{1}}$.

Definition 3. We propose that the inverse Fourier transform of the complex function (similar to Eq. (3))

$$
\frac{1}{(-2 \pi i)^{2}} \frac{\operatorname{sgn} \sigma}{\omega^{0} \omega^{1}}
$$


is equal to 1. In other words, we propose that the inverse Fourier transform of $1 /\left(\omega^{0} \omega^{1}\right), \mathcal{F}^{-1}\left[1 /\left(\omega^{0} \omega^{1}\right)\right]$ is equal to $(-2 \pi i)^{2}$ sgno because $(\operatorname{sgn} \sigma)^{2}=1$ :

$$
\begin{aligned}
\mathcal{F}^{-1}\left[\frac{1}{\omega^{0} \omega^{1}}\right] & :=\lim _{\varepsilon \rightarrow 0}(-2 \pi i)^{2} \varepsilon^{2} \int_{\Gamma_{\sigma}}\left(\omega^{0}\right)^{-1+\varepsilon}\left(\omega^{1}\right)^{-1+\varepsilon} e^{\omega^{0} \bar{\pi}_{0}+\omega^{1} \bar{\pi}_{1}} d \omega^{0} d \omega^{1} \\
& =(-2 \pi i)^{2} \operatorname{sgn} \sigma, \quad\left(\bar{\pi}_{0}, \bar{\pi}_{1}\right) \in-\Gamma_{\sigma}+i \mathbb{R}^{2} .
\end{aligned}
$$

When we take $\sigma=(1,-1)$, the integral in Eq. (12) represents an improper integral from 0 to $\infty$ on the real axis for the variable $\omega^{0}$ and from $-\infty$ to 0 on the real axis for the variable $\omega^{1}$ :

$$
\begin{aligned}
& \lim _{\varepsilon \rightarrow 0}(-2 \pi i)^{2} \varepsilon^{2} \int_{\Gamma_{(1,-1)}}\left(\omega^{0}\right)^{-1+\varepsilon}\left(\omega^{1}\right)^{-1+\varepsilon} e^{\omega^{0} \bar{\pi}_{0}+\omega^{1} \bar{\pi}_{1}} d \omega^{0} d \omega^{1} \\
= & \lim _{\varepsilon \rightarrow 0}(-2 \pi i)^{2} \varepsilon^{2} \int_{0}^{\infty}\left(\operatorname{Re} \omega^{0}\right)^{-1+\varepsilon} e^{\left(\operatorname{Re} \omega^{0}\right) \bar{\pi}_{0}} d\left(\operatorname{Re} \omega^{0}\right) \\
& \times \int_{-\infty}^{0}\left(\operatorname{Re} \omega^{1}\right)^{-1+\varepsilon} e^{\left(\operatorname{Re} \omega^{1}\right) \bar{\pi}_{1}} d\left(\operatorname{Re} \omega^{1}\right) \\
= & (-2 \pi i)^{2}(-1), \quad\left(\bar{\pi}_{0}, \bar{\pi}_{1}\right) \in-\Gamma_{(1,-1)}+i \mathbb{R}^{2}
\end{aligned}
$$

Here, the integration formula

$$
\int_{0}^{\infty} x^{z-1+\varepsilon} e^{-w x} d x=\frac{\Gamma(z+\varepsilon)}{w^{z+\varepsilon}}, \quad \operatorname{Re} w>0
$$

and the gamma function formula

$$
\Gamma(\varepsilon)=\frac{1}{\varepsilon}-\gamma+\mathcal{O}(\varepsilon)
$$

have been used, where $\gamma$ is the Euler-Mascheroni constant. For all other $\sigma$, the meaning of the integral sign in Eq. (12) is the same, and the integral is uniformly convergent on $\left(\bar{\pi}_{0}, \bar{\pi}_{1}\right) \in-\Gamma_{\sigma}+i \mathbb{R}^{2}$.

Theorem 4. The inverse Fourier transform of the twistor-space function $\tilde{f}\left(\omega^{\alpha}, \pi_{\dot{\alpha}}\right)$ is

$$
\begin{aligned}
& \mathcal{F}^{-1}\left[\tilde{f}\left(\omega^{\alpha}, \pi_{\dot{\alpha}}\right)\right] \\
& :=\lim _{\varepsilon \rightarrow 0}(-2 \pi i)^{2} \varepsilon^{2} \int_{\Gamma_{\sigma}} \tilde{f}\left(\omega^{\alpha}, \pi_{\dot{\alpha}}\right)\left(\omega^{0}\right)^{\varepsilon}\left(\omega^{1}\right)^{\varepsilon} e^{\omega^{0} \bar{\pi}_{0}+\omega^{1} \bar{\pi}_{1}} d \omega^{0} d \omega^{1} \\
& =\sum_{a, b=0}^{\infty} D_{a b}\left(\pi_{\dot{0}}, \pi_{\dot{1}}\right)(-2 \pi i)^{2} \operatorname{sgn} \sigma \frac{\left(\bar{\pi}_{0}\right)^{a}\left(\bar{\pi}_{1}\right)^{b}}{\Gamma(a+1) \Gamma(b+1)} \\
& \left(\bar{\pi}_{0}, \bar{\pi}_{1}\right) \in-\Gamma_{\sigma}+i \mathbb{R}^{2} .
\end{aligned}
$$

By using the analytic continuation method, we can extend the $\left\{\left(\bar{\pi}_{0}, \bar{\pi}_{1}\right)\right\}$ region from $-\Gamma_{\sigma}+i \mathbb{R}^{2}$ to $\mathbb{C}^{2}$. Hereafter, we consider the $\left\{\left(\bar{\pi}_{0}, \bar{\pi}_{1}\right)\right\}$ region in Eq. (16) as $\mathbb{C}^{2}$. 
Proof. From Eq. (12), the inverse Fourier transform of the power series term $\left(\omega^{0}\right)^{-a-1}\left(\omega^{1}\right)^{-b-1}(a, b=0,1,2, \cdots)$ is

$$
\begin{aligned}
& \mathcal{F}^{-1}\left[\left(\omega^{0}\right)^{-a-1}\left(\omega^{1}\right)^{-b-1}\right] \\
& :=\lim _{\varepsilon \rightarrow 0}(-2 \pi i)^{2} \varepsilon^{2} \int_{\Gamma_{\sigma}}\left(\omega^{0}\right)^{-a-1+\varepsilon}\left(\omega^{1}\right)^{-b-1+\varepsilon} e^{\omega^{0} \bar{\pi}_{0}+\omega^{1} \bar{\pi}_{1}} d \omega^{0} d \omega^{1} \\
& =(-2 \pi i)^{2} \operatorname{sgn} \sigma \frac{\left(\bar{\pi}_{0}\right)^{a}\left(\bar{\pi}_{1}\right)^{b}}{\Gamma(a+1) \Gamma(b+1)}, \quad\left(\bar{\pi}_{0}, \bar{\pi}_{1}\right) \in-\Gamma_{\sigma}+i \mathbb{R}^{2} .
\end{aligned}
$$

The integral in Eq. (17) can be calculated in the same way as that in Eq. (12). Here, we have used the integration formula in Eq. (14) and the gamma function formula

$$
\Gamma(-n+\varepsilon)=\frac{(-1)^{n}}{n !}\left[\frac{1}{\varepsilon}+\psi_{1}(n+1)+\mathcal{O}(\varepsilon)\right], \quad n=0,1,2, \cdots,
$$

where $\psi_{1}(n+1):=\Gamma^{\prime}(n+1) / \Gamma(n+1)=\sum_{p=1}^{n} p^{-1}-\gamma$. Thus, from Eqs. (10) and (17), Eq. (16) is obtained.

Corollary 5. The inverse Fourier transform in Eq. (16) is precisely the inverse map of the Fourier transform in Eq. (7), i.e.,

$$
\mathcal{F}^{-1} \mathcal{F}\left[f\left(\bar{\pi}_{\alpha}, \pi_{\dot{\alpha}}\right)\right]=f\left(\bar{\pi}_{\alpha}, \pi_{\dot{\alpha}}\right), \quad \mathcal{F F}^{-1}\left[\tilde{f}\left(\omega^{\alpha}, \pi_{\dot{\alpha}}\right)\right]=\tilde{f}\left(\omega^{\alpha}, \pi_{\dot{\alpha}}\right) .
$$

Proof. From Eqs. (8) and (17), we have

$$
\begin{aligned}
\mathcal{F}^{-1} \mathcal{F}\left[\left(\bar{\pi}_{0}\right)^{a}\left(\bar{\pi}_{1}\right)^{b}\right] & =\mathcal{F}^{-1}\left[\frac{\operatorname{sgn} \sigma}{(-2 \pi i)^{2}} \frac{\Gamma(a+1) \Gamma(b+1)}{\left(\omega^{0}\right)^{a+1}\left(\omega^{1}\right)^{b+1}}\right] \\
= & \left(\bar{\pi}_{0}\right)^{a}\left(\bar{\pi}_{1}\right)^{b}, \\
\mathcal{F F}^{-1}\left[\left(\omega^{0}\right)^{-a-1}\left(\omega^{1}\right)^{-b-1}\right] & =\mathcal{F}\left[(-2 \pi i)^{2} \operatorname{sgn} \sigma \frac{\left(\bar{\pi}_{0}\right)^{a}\left(\bar{\pi}_{1}\right)^{b}}{\Gamma(a+1) \Gamma(b+1)}\right] \\
& =\left(\omega^{0}\right)^{-a-1}\left(\omega^{1}\right)^{-b-1} .
\end{aligned}
$$

Thus, from Eqs. (4), (10), (20), and (21), we obtain Eq. (19).

\section{Cohomological interpretation}

\subsection{Brief review of Čech cohomology group}

We now consider the one-dimensional complex projective space $\mathbb{C P}^{1}$ covered by the two open sets

$$
U_{0}=\left\{\left(\bar{\pi}_{0}, \bar{\pi}_{1}\right) \in \mathbb{C P}^{1} \mid \bar{\pi}_{0} \neq 0\right\}, \quad U_{1}=\left\{\left(\bar{\pi}_{0}, \bar{\pi}_{1}\right) \in \mathbb{C P}^{1} \mid \bar{\pi}_{1} \neq 0\right\} .
$$

Let $\mathcal{O}(n)(n \in \mathbb{Z})$ be the sheaf of germs of homogeneous holomorphic functions on $\mathbb{C P}^{1}$ of degree $n$. Then, the zeroth Cech cohomology group on $\mathbb{C P}^{1}$ with coefficients 
Jun-ichi Note

in the sheaf $\mathcal{O}(n)$ is represented as [16.

$$
\begin{aligned}
& H^{0}\left(\mathbb{C P}^{1}, \mathcal{O}(n)\right) \\
& =\left\{\sum_{r=1}^{n+1} a_{r}\left(\bar{\pi}_{0}\right)^{r-1}\left(\bar{\pi}_{1}\right)^{n+1-r} \mid a_{r} \in \mathbb{C}\right\}, \quad n=0,1,2, \cdots .
\end{aligned}
$$

This vanishes for negative integers $n=-1,-2,-3, \cdots$.

When $\mathbb{C P}^{1}$ is covered by the open sets

$$
U_{0}^{\prime}=\left\{\left(\omega^{0}, \omega^{1}\right) \in \mathbb{C P}^{1} \mid \omega^{0} \neq 0\right\}, \quad U_{1}^{\prime}=\left\{\left(\omega^{0}, \omega^{1}\right) \in \mathbb{C P}^{1} \mid \omega^{1} \neq 0\right\},
$$

the first Čech cohomology group on $\mathbb{C P}^{1}$ with coefficients in the sheaf $\mathcal{O}(-n-2)$ is represented as 16

$$
\begin{aligned}
& H^{1}\left(\mathbb{C P}^{1}, \mathcal{O}(-n-2)\right) \\
& =\left\{\sum_{r=1}^{n+1} \frac{a_{r}}{\left(\omega^{0}\right)^{r}\left(\omega^{1}\right)^{n+2-r}} \mid a_{r} \in \mathbb{C}\right\}, \quad n=0,1,2, \cdots .
\end{aligned}
$$

This also vanishes for negative integers $n=-1,-2,-3, \cdots$. The elements of $H^{1}\left(\mathbb{C P}^{1}, \mathcal{O}(-n-2)\right)$ are equivalent to elements with extra homogeneous holomorphic functions on $U_{i}^{\prime}(i=0,1)$ of degree $(-n-2)$ [16]:

$$
\begin{aligned}
\sum_{r=1}^{n+1} \frac{a_{r}}{\left(\omega^{0}\right)^{r}\left(\omega^{1}\right)^{n+2-r}} & \sim \sum_{r=1}^{n+1} \frac{a_{r}}{\left(\omega^{0}\right)^{r}\left(\omega^{1}\right)^{n+2-r}} \\
& +\sum_{s=0}^{\infty}\left(b_{s} \frac{\left(\omega^{1}\right)^{s}}{\left(\omega^{0}\right)^{n+2+s}}+c_{s} \frac{\left(\omega^{0}\right)^{s}}{\left(\omega^{1}\right)^{n+2+s}}\right) .
\end{aligned}
$$

When $n=0,1,2, \cdots$, the Čech cohomology groups in Eqs. (23) and (25) are isomorphic to $\mathbb{C}^{n+1}$. However, they are not the same; instead, they are dual spaces of each other. This is a particular instance of Serre duality [17.

Now, let $L$ be a complex projective line in the twistor space $\mathbb{P} \mathbb{T}$ defined as $L=\left\{\left(\omega^{\alpha}, \pi_{\dot{\alpha}}\right) \in \mathbb{P} \mathbb{T} \mid \omega^{0}=\omega^{1}=0\right\} \simeq \mathbb{C P}^{1}$. Let $\mathbb{P} \mathbb{T}-L$ be covered by

$$
V_{0}=\left\{\left(\omega^{\alpha}, \pi_{\dot{\alpha}}\right) \in \mathbb{P} \mathbb{T} \mid \omega^{0} \neq 0\right\}, \quad V_{1}=\left\{\left(\omega^{\alpha}, \pi_{\dot{\alpha}}\right) \in \mathbb{P} \mathbb{T} \mid \omega^{1} \neq 0\right\} .
$$

The first Čech cohomology group on $\mathbb{P} \mathbb{T}-L$ with coefficients in the sheaf $\mathcal{O}(m)$ $(m \in \mathbb{Z})$ is represented as 18 ]

$$
H^{1}(\mathbb{P} \mathbb{T}-L, \mathcal{O}(m))=\left\{\sum_{j, k>0} \frac{A_{j k}\left(\pi_{\dot{0}}, \pi_{\dot{1}}\right)}{\left(\omega^{0}\right)^{j}\left(\omega^{1}\right)^{k}}\right\} .
$$

Here, $A_{j k}\left(\pi_{\dot{0}}, \pi_{\dot{1}}\right)$ denotes the homogeneous holomorphic function on $L$ of degree $(j+k+m): A_{j k}\left(\pi_{\dot{0}}, \pi_{\dot{1}}\right) \in \Gamma(L, \mathcal{O}(j+k+m))=H^{0}(L, \mathcal{O}(j+k+m))$. Hence, the sum in Eq. (28) is taken for $j>0$ and $k>0$ satisfying $j+k+m \geq 0$, because $H^{0}(L, \mathcal{O}(j+k+m))$ is 0 for $j+k+m<0$.

We emphasize that Eq. (28) is precisely the twistor-space functions to which the inverse Fourier transform is applied, as seen from Eq. (10). In the twistor theory 
literature, the elements of Eq. (28) are referred to as elementary states [19, and they are used in the calculation of twistor diagrams [13].

\subsection{New theorem and its corollary}

Hereafter, we identify the properties of the Fourier transform in terms of Cech cohomology groups.

Theorem 6. The Fourier transform is an isomorphism from $H^{0}\left(\mathbb{C P}^{1}, \mathcal{O}(n)\right)$ to $H^{1}\left(\mathbb{C P}^{1}, \mathcal{O}(-n-2)\right)$, and the inverse Fourier transform is an isomorphism from $H^{1}\left(\mathbb{C P}^{1}, \mathcal{O}(-n-2)\right)$ to $H^{0}\left(\mathbb{C P}^{1}, \mathcal{O}(n)\right)$.

Proof. From Eq. (8), the Fourier transform of the basis $\left\{\left(\bar{\pi}_{0}\right)^{r-1}\left(\bar{\pi}_{1}\right)^{n+1-r}\right\}$ of $H^{0}\left(\mathbb{C P}^{1}, \mathcal{O}(n)\right)$ is

$$
\mathcal{F}\left[\left(\bar{\pi}_{0}\right)^{r-1}\left(\bar{\pi}_{1}\right)^{n+1-r}\right]=\frac{\operatorname{sgn} \sigma}{(-2 \pi i)^{2}} \frac{\Gamma(r) \Gamma(n+2-r)}{\left(\omega^{0}\right)^{r}\left(\omega^{1}\right)^{n+2-r}} .
$$

Equation (29) is precisely the basis $\left\{\left(\omega^{0}\right)^{-r}\left(\omega^{1}\right)^{-n-2+r}\right\}$ of $H^{1}\left(\mathbb{C P}^{1}, \mathcal{O}(-n-\right.$ 2)). Furthermore, from Eq. (17), the inverse Fourier transform of the basis $\left\{\left(\omega^{0}\right)^{-r}\left(\omega^{1}\right)^{-n-2+r}\right\}$ of $H^{1}\left(\mathbb{C P}^{1}, \mathcal{O}(-n-2)\right)$ is

$$
\mathcal{F}^{-1}\left[\left(\omega^{0}\right)^{-r}\left(\omega^{1}\right)^{-n-2+r}\right]=(-2 \pi i)^{2} \operatorname{sgn} \sigma \frac{\left(\bar{\pi}_{0}\right)^{r-1}\left(\bar{\pi}_{1}\right)^{n+1-r}}{\Gamma(r) \Gamma(n+2-r)} .
$$

In addition, if we take the inverse Fourier transform of a homogeneous holomorphic function on $U_{i}^{\prime}(i=0,1)$ of degree $(-n-2)$, which is added to the equivalence class of $H^{1}\left(\mathbb{C P}^{1}, \mathcal{O}(-n-2)\right)$, i.e., $\left(\omega^{1}\right)^{s} /\left(\omega^{0}\right)^{n+2+s}$ or $\left(\omega^{0}\right)^{s} /\left(\omega^{1}\right)^{n+2+s}(s=0,1,2, \cdots)$, we obtain 0 because the gamma function in the denominator diverges:

$$
\mathcal{F}^{-1}\left[\left(\omega^{1}\right)^{s} /\left(\omega^{0}\right)^{n+2+s}\right]=\mathcal{F}^{-1}\left[\left(\omega^{0}\right)^{s} /\left(\omega^{1}\right)^{n+2+s}\right]=0 .
$$

Therefore, the inverse Fourier transform of the basis $\left\{\left(\omega^{0}\right)^{-r}\left(\omega^{1}\right)^{-n-2+r}\right\}$ of $H^{1}\left(\mathbb{C P}^{1}, \mathcal{O}(-n-2)\right)$ is precisely the basis $\left\{\left(\bar{\pi}_{0}\right)^{r-1}\left(\bar{\pi}_{1}\right)^{n+1-r}\right\}$ of $H^{0}\left(\mathbb{C P}^{1}, \mathcal{O}(n)\right) \square$

We emphasize that the mapping composed of the inverse Fourier transform and the change of variables $\left(\bar{\pi}_{0}, \bar{\pi}_{1}\right) \mapsto\left(\omega^{0}, \omega^{1}\right)$ is an isomorphism from $H^{1}\left(\mathbb{C P}^{1}, \mathcal{O}(-n-\right.$ 2)) to its dual space $H^{0}\left(\mathbb{C P}^{1}, \mathcal{O}(n)\right)$.

Corollary 7. The momentum-space functions to which the Fourier transform is applied are represented as

$$
\bigoplus_{n=0}^{\infty} H^{0}(L, \mathcal{O}(n+2+m)) \otimes H^{0}\left(\mathbb{C P}^{1}, \mathcal{O}(n)\right)
$$

Proof. Let us consider subsets of the basis of Eq. (28) that satisfy the condition $j+k=n+2(n=0,1,2, \cdots)$. The elements of these subsets are products of 


$$
\begin{aligned}
A_{j k}\left(\pi_{\dot{0}}, \pi_{\dot{1}}\right) \in H^{0}(L, \mathcal{O}(n+2+m)) \text { and } 1 /\left(\omega^{0}\right)^{j}\left(\omega^{1}\right)^{k} \in H^{1}\left(\mathbb{C P}^{1}, \mathcal{O}(-n-2)\right), \text { i.e., } \\
\left.\qquad \sum_{\substack{j, k>0 \\
j+k=n+2}} \frac{A_{j k}\left(\pi_{\dot{0}}, \pi_{\dot{1}}\right)}{\left(\omega^{0}\right)^{j}\left(\omega^{1}\right)^{k}}\right\} \\
=H^{0}(L, \mathcal{O}(n+2+m)) \otimes H^{1}\left(\mathbb{C P}^{1}, \mathcal{O}(-n-2)\right) .
\end{aligned}
$$

Therefore, from Eqs. (28) and (33),

$$
\begin{aligned}
& H^{1}(\mathbb{P} \mathbb{T}-L, \mathcal{O}(m)) \\
& =\bigoplus_{n=0}^{\infty} H^{0}(L, \mathcal{O}(n+2+m)) \otimes H^{1}\left(\mathbb{C P}^{1}, \mathcal{O}(-n-2)\right) .
\end{aligned}
$$

Because the inverse Fourier transform is an isomorphism from $H^{1}\left(\mathbb{C P}^{1}, \mathcal{O}(-n-2)\right)$ to $H^{0}\left(\mathbb{C P}^{1}, \mathcal{O}(n)\right)$, taking the inverse Fourier transform of Eq. (34) gives Eq. (32)

\section{Twistor operator representations}

\subsection{Background}

In the quantization procedure in twistor theory, the variables $\omega^{\alpha}$ and $\bar{\pi}_{\alpha}$ become the operators $\hat{\omega}^{\alpha}$ and $\hat{\bar{\pi}}_{\alpha}$ satisfying the commutation relations [12]13]14

$$
\left[\hat{\omega}^{\alpha}, \hat{\bar{\pi}}_{\beta}\right]=\delta_{\beta}^{\alpha}, \quad\left[\hat{\omega}^{\alpha}, \hat{\omega}^{\beta}\right]=0, \quad\left[\hat{\bar{\pi}}_{\alpha}, \hat{\bar{\pi}}_{\beta}\right]=0 .
$$

By analogy with standard quantum mechanics, in the representation that diagonalizes $\hat{\bar{\pi}}_{\alpha}$, these operators are represented as

$$
\hat{\bar{\pi}}_{\alpha} \doteq \bar{\pi}_{\alpha}, \quad \hat{\omega}^{\alpha} \doteq \frac{\partial}{\partial \bar{\pi}_{\alpha}} .
$$

Here, the symbol $\doteq$ stands for "is represented by." In addition, in the representation that diagonalizes $\hat{\omega}^{\alpha}$,

$$
\hat{\bar{\pi}}_{\alpha} \doteq-\frac{\partial}{\partial \omega^{\alpha}}, \quad \hat{\omega}^{\alpha} \doteq \omega^{\alpha} .
$$

Therefore, the following correspondence relations between elements $f\left(\bar{\pi}_{\alpha}, \pi_{\dot{\alpha}}\right)$ of Eq. (32) and their Fourier transforms $\mathcal{F}\left[f\left(\bar{\pi}_{\alpha}, \pi_{\dot{\alpha}}\right)\right]=\tilde{f}\left(\omega^{\alpha}, \pi_{\dot{\alpha}}\right)$ are desirable:

$$
\begin{gathered}
\bar{\pi}_{\alpha} f\left(\bar{\pi}_{\alpha}, \pi_{\dot{\alpha}}\right) \leftrightarrow-\frac{\partial}{\partial \omega^{\alpha}} \tilde{f}\left(\omega^{\alpha}, \pi_{\dot{\alpha}}\right), \\
\frac{\partial}{\partial \bar{\pi}_{\alpha}} f\left(\bar{\pi}_{\alpha}, \pi_{\dot{\alpha}}\right) \leftrightarrow \omega^{\alpha} \tilde{f}\left(\omega^{\alpha}, \pi_{\dot{\alpha}}\right) .
\end{gathered}
$$

In Sections 4.2 and 4.3 , we show that such correspondence relations exist, and in Section 4.4, we identify the functions over which the operators $\hat{\omega}^{\alpha}$ and $\hat{\bar{\pi}}_{\alpha}$ are closed. 


\subsection{Fourier transform of the operators}

Theorem 8. The correspondence relations in Eqs. (38) and (39) are satisfied by the Fourier transform, i.e.,

$$
\begin{aligned}
& \mathcal{F}\left[\bar{\pi}_{\alpha} f\left(\bar{\pi}_{\alpha}, \pi_{\dot{\alpha}}\right)\right]=-\frac{\partial}{\partial \omega^{\alpha}} \mathcal{F}\left[f\left(\bar{\pi}_{\alpha}, \pi_{\dot{\alpha}}\right)\right], \\
& \mathcal{F}\left[\frac{\partial}{\partial \bar{\pi}_{\alpha}} f\left(\bar{\pi}_{\alpha}, \pi_{\dot{\alpha}}\right)\right]=\omega^{\alpha} \mathcal{F}\left[f\left(\bar{\pi}_{\alpha}, \pi_{\dot{\alpha}}\right)\right] .
\end{aligned}
$$

Proof. For the basis $\left\{\left(\bar{\pi}_{0}\right)^{r-1}\left(\bar{\pi}_{1}\right)^{n+1-r}\right\}_{r=1}^{n+1}$ of $H^{0}\left(\mathbb{C P}^{1}, \mathcal{O}(n)\right)$, it is seen from Eq. (8) that

$$
\begin{gathered}
\mathcal{F}\left[\bar{\pi}_{0}\left(\bar{\pi}_{0}\right)^{r-1}\left(\bar{\pi}_{1}\right)^{n+1-r}\right]=\frac{\operatorname{sgn} \sigma}{(-2 \pi i)^{2}} \frac{\Gamma(r+1) \Gamma(n+2-r)}{\left(\omega^{0}\right)^{r+1}\left(\omega^{1}\right)^{n+2-r}}, \\
-\frac{\partial}{\partial \omega^{0}} \mathcal{F}\left[\left(\bar{\pi}_{0}\right)^{r-1}\left(\bar{\pi}_{1}\right)^{n+1-r}\right]=\frac{\operatorname{sgn} \sigma}{(-2 \pi i)^{2}} \frac{r \Gamma(r) \Gamma(n+2-r)}{\left(\omega^{0}\right)^{r+1}\left(\omega^{1}\right)^{n+2-r}} .
\end{gathered}
$$

Hence, we have

$$
\mathcal{F}\left[\bar{\pi}_{0}\left(\bar{\pi}_{0}\right)^{r-1}\left(\bar{\pi}_{1}\right)^{n+1-r}\right]=-\frac{\partial}{\partial \omega^{0}} \mathcal{F}\left[\left(\bar{\pi}_{0}\right)^{r-1}\left(\bar{\pi}_{1}\right)^{n+1-r}\right] .
$$

Furthermore, it is seen from Eq. (8) that

$$
\begin{aligned}
& \mathcal{F}\left[\frac{\partial}{\partial \bar{\pi}_{0}}\left(\bar{\pi}_{0}\right)^{r-1}\left(\bar{\pi}_{1}\right)^{n+1-r}\right] \\
& = \begin{cases}\frac{\operatorname{sgn} \sigma}{(-2 \pi i)^{2}} \frac{(r-1) \Gamma(r-1) \Gamma(n+2-r)}{\left(\omega^{0}\right)^{r-1}\left(\omega^{1}\right)^{n+2-r}} & (r \neq 1) \\
0 & (r=1)\end{cases} \\
& \omega^{0} \mathcal{F}\left[\left(\bar{\pi}_{0}\right)^{r-1}\left(\bar{\pi}_{1}\right)^{n+1-r}\right]=\frac{\operatorname{sgn} \sigma}{(-2 \pi i)^{2}} \frac{\Gamma(r) \Gamma(n+2-r)}{\left(\omega^{0}\right)^{r-1}\left(\omega^{1}\right)^{n+2-r}} .
\end{aligned}
$$

When $r=1$, Eq. (46) becomes

$$
\frac{\operatorname{sgn} \sigma}{(-2 \pi i)^{2}} \frac{\Gamma(n+1)}{\left(\omega^{1}\right)^{n+1}}
$$

This is equivalent to 0 as the element of $H^{1}\left(\mathbb{C P}^{1}, \mathcal{O}(-n-1)\right)$. Hence, we have

$$
\mathcal{F}\left[\frac{\partial}{\partial \bar{\pi}_{0}}\left(\bar{\pi}_{0}\right)^{r-1}\left(\bar{\pi}_{1}\right)^{n+1-r}\right]=\omega^{0} \mathcal{F}\left[\left(\bar{\pi}_{0}\right)^{r-1}\left(\bar{\pi}_{1}\right)^{n+1-r}\right] .
$$

We can also obtain equations similar to Eqs. (44) and (48) for the variables $\bar{\pi}_{1}$ and $\omega^{1}$. Hence, for the elements $f\left(\bar{\pi}_{\alpha}, \pi_{\dot{\alpha}}\right)$ of Eq. (32), we obtain Eqs. (40) and (41). $\square$ 


\subsection{Inverse Fourier transform of the operators}

Theorem 9. The correspondence relations in Eqs. (38) and (39) are satisfied by the inverse Fourier transform, i.e.,

$$
\begin{gathered}
\mathcal{F}^{-1}\left[-\frac{\partial}{\partial \omega^{\alpha}} \tilde{f}\left(\omega^{\alpha}, \pi_{\dot{\alpha}}\right)\right]=\bar{\pi}_{\alpha} \mathcal{F}^{-1}\left[\tilde{f}\left(\omega^{\alpha}, \pi_{\dot{\alpha}}\right)\right], \\
\mathcal{F}^{-1}\left[\omega^{\alpha} \tilde{f}\left(\omega^{\alpha}, \pi_{\dot{\alpha}}\right)\right]=\frac{\partial}{\partial \bar{\pi}_{\alpha}} \mathcal{F}^{-1}\left[\tilde{f}\left(\omega^{\alpha}, \pi_{\dot{\alpha}}\right)\right] .
\end{gathered}
$$

Proof. For the basis $\left\{\left(\omega^{0}\right)^{-r}\left(\omega^{1}\right)^{-n-2+r}\right\}_{r=1}^{n+1}$ of $H^{1}\left(\mathbb{C P}^{1}, \mathcal{O}(-n-2)\right)$, it is seen from Eq. (17) that

$$
\begin{gathered}
\mathcal{F}^{-1}\left[-\frac{\partial}{\partial \omega^{0}}\left(\omega^{0}\right)^{-r}\left(\omega^{1}\right)^{-n-2+r}\right]=(-2 \pi i)^{2} \operatorname{sgn} \sigma \frac{r\left(\bar{\pi}_{0}\right)^{r}\left(\bar{\pi}_{1}\right)^{n+1-r}}{\Gamma(r+1) \Gamma(n+2-r)}, \\
\bar{\pi}_{0} \mathcal{F}^{-1}\left[\left(\omega^{0}\right)^{-r}\left(\omega^{1}\right)^{-n-2+r}\right]=(-2 \pi i)^{2} \operatorname{sgn} \sigma \frac{\left(\bar{\pi}_{0}\right)^{r}\left(\bar{\pi}_{1}\right)^{n+1-r}}{\Gamma(r) \Gamma(n+2-r)} .
\end{gathered}
$$

Hence, we have

$$
\mathcal{F}^{-1}\left[-\frac{\partial}{\partial \omega^{0}}\left(\omega^{0}\right)^{-r}\left(\omega^{1}\right)^{-n-2+r}\right]=\bar{\pi}_{0} \mathcal{F}^{-1}\left[\left(\omega^{0}\right)^{-r}\left(\omega^{1}\right)^{-n-2+r}\right] .
$$

Furthermore, it is seen from Eq. (17) that

$$
\begin{gathered}
\mathcal{F}^{-1}\left[\omega^{0}\left(\omega^{0}\right)^{-r}\left(\omega^{1}\right)^{-n-2+r}\right] \\
= \begin{cases}(-2 \pi i)^{2} \operatorname{sgn} \sigma \frac{\left(\bar{\pi}_{0}\right)^{r-2}\left(\bar{\pi}_{1}\right)^{n+1-r}}{\Gamma(r-1) \Gamma(n+2-r)} & (r \neq 1) \\
0 & (r=1)\end{cases} \\
\quad \frac{\partial}{\partial \bar{\pi}_{0}} \mathcal{F}^{-1}\left[\left(\omega^{0}\right)^{-r}\left(\omega^{1}\right)^{-n-2+r}\right] \\
=(-2 \pi i)^{2} \operatorname{sgn} \sigma \frac{(r-1)\left(\bar{\pi}_{0}\right)^{r-2}\left(\bar{\pi}_{1}\right)^{n+1-r}}{\Gamma(r) \Gamma(n+2-r)} .
\end{gathered}
$$

Hence, we have

$$
\mathcal{F}^{-1}\left[\omega^{0}\left(\omega^{0}\right)^{-r}\left(\omega^{1}\right)^{-n-2+r}\right]=\frac{\partial}{\partial \bar{\pi}_{0}} \mathcal{F}^{-1}\left[\left(\omega^{0}\right)^{-r}\left(\omega^{1}\right)^{-n-2+r}\right] .
$$

We can also obtain equations similar to Eqs. (53) and (56) for the variables $\omega^{1}$ and $\bar{\pi}_{1}$. Hence, for the elements $\tilde{f}\left(\omega^{\alpha}, \pi_{\dot{\alpha}}\right)$ of Eq. (28), we obtain Eqs. (49) and (50). 


\subsection{Applying the operators}

Theorem 10. The application of the operators $\hat{\bar{\pi}}_{\alpha} \doteq \bar{\pi}_{\alpha}$ and $\hat{\omega}^{\alpha} \doteq \partial / \partial \bar{\pi}_{\alpha}$ is closed on the momentum-space functions

$$
\bigoplus_{m=-\infty}^{\infty} \bigoplus_{n=0}^{\infty} H^{0}(L, \mathcal{O}(n+2+m)) \otimes H^{0}\left(\mathbb{C P}^{1}, \mathcal{O}(n)\right)
$$

to which the Fourier transform is applied.

Proof. By applying the multiplication operators $\hat{\bar{\pi}}_{\alpha} \doteq \bar{\pi}_{\alpha}$ to the basis $\left\{\left(\bar{\pi}_{0}\right)^{r-1}\left(\bar{\pi}_{1}\right)^{n+1-r}\right\}_{r=1}^{n+1}$ of $H^{0}\left(\mathbb{C P}^{1}, \mathcal{O}(n)\right)$, for $n=0,1,2, \cdots$, we have

$$
\bar{\pi}_{\alpha}: H^{0}\left(\mathbb{C P}^{1}, \mathcal{O}(n)\right) \rightarrow H^{0}\left(\mathbb{C P}^{1}, \mathcal{O}(n+1)\right) .
$$

In addition, applying the partial differential operator $\hat{\omega}^{0} \doteq \partial / \partial \bar{\pi}_{0}$ gives

$$
\frac{\partial}{\partial \bar{\pi}_{0}}:\left(\bar{\pi}_{0}\right)^{r-1}\left(\bar{\pi}_{1}\right)^{n+1-r} \mapsto(r-1)\left(\bar{\pi}_{0}\right)^{r-2}\left(\bar{\pi}_{1}\right)^{n+1-r} .
$$

When $r=1$, the result is 0 , and the same is true for the partial differential operator $\hat{\omega}^{1} \doteq \partial / \partial \bar{\pi}_{1}$. Thus, for $n=0$, we have

$$
\frac{\partial}{\partial \bar{\pi}_{\alpha}}: H^{0}\left(\mathbb{C P}^{1}, \mathcal{O}(0)\right) \rightarrow 0,
$$

and for $n=1,2,3, \cdots$, we have

$$
\frac{\partial}{\partial \bar{\pi}_{\alpha}}: H^{0}\left(\mathbb{C P}^{1}, \mathcal{O}(n)\right) \rightarrow H^{0}\left(\mathbb{C P}^{1}, \mathcal{O}(n-1)\right) .
$$

Thus, the application of the operators is closed on Eq. (57).

Theorem 11. The application of the operators $\hat{\bar{\pi}}_{\alpha} \doteq-\partial / \partial \omega^{\alpha}$ and $\hat{\omega}^{\alpha} \doteq \omega^{\alpha}$ is closed on the twistor-space functions

$$
\begin{aligned}
& \bigoplus_{m=-\infty}^{\infty} H^{1}(\mathbb{P} \mathbb{T}-L, \mathcal{O}(m)) \\
= & \bigoplus_{m=-\infty}^{\infty} \bigoplus_{n=0}^{\infty} H^{0}(L, \mathcal{O}(n+2+m)) \otimes H^{1}\left(\mathbb{C P}^{1}, \mathcal{O}(-n-2)\right),
\end{aligned}
$$

to which the inverse Fourier transform is applied.

Proof. By applying the partial differential operators $\hat{\bar{\pi}}_{\alpha} \doteq-\partial / \partial \omega^{\alpha}$ to the basis $\left\{\left(\omega^{0}\right)^{-r}\left(\omega^{1}\right)^{-n-2+r}\right\}_{r=1}^{n+1}$ of $H^{1}\left(\mathbb{C P}^{1}, \mathcal{O}(-n-2)\right)$, for $n=0,1,2, \cdots$, we have

$$
-\frac{\partial}{\partial \omega^{\alpha}}: H^{1}\left(\mathbb{C P}^{1}, \mathcal{O}(-n-2)\right) \rightarrow H^{1}\left(\mathbb{C P}^{1}, \mathcal{O}(-n-3)\right) .
$$

In addition, applying the multiplication operator $\hat{\omega}^{0} \doteq \omega^{0}$ gives

$$
\omega^{0}:\left(\omega^{0}\right)^{-r}\left(\omega^{1}\right)^{-n-2+r} \mapsto\left(\omega^{0}\right)^{-r+1}\left(\omega^{1}\right)^{-n-2+r} .
$$


When $r=1$, we have $\left(\omega^{1}\right)^{-n-1}$. This is equivalent to 0 as the element of $H^{1}\left(\mathbb{C P}^{1}, \mathcal{O}(-n-1)\right)$. The same is true for the multiplication operator $\hat{\omega}^{1} \doteq \omega^{1}$. Hence, for $n=0$, we have

$$
\omega^{\alpha}: H^{1}\left(\mathbb{C P}^{1}, \mathcal{O}(-2)\right) \rightarrow 0,
$$

and for $n=1,2,3, \cdots$, we have

$$
\omega^{\alpha}: H^{1}\left(\mathbb{C P}^{1}, \mathcal{O}(-n-2)\right) \rightarrow H^{1}\left(\mathbb{C P}^{1}, \mathcal{O}(-n-1)\right) .
$$

From this fact and Eq. (34), the application of the operators is closed on Eq. (62)

\section{Summary and discussion}

We proposed a new definition of the Fourier transform from momentum space to twistor space as well as its inverse. Here, the momentum-space coordinates are defined by the $S L(2, \mathbb{C})$ spinor $\left(\pi_{\dot{\alpha}}\right)_{\dot{\alpha}=\dot{0}, \dot{1}}$ and its complex conjugate $\left(\bar{\pi}_{\alpha}\right)_{\alpha=0,1}$, and the twistor-space coordinates are defined by the $S L(2, \mathbb{C})$ spinors $\left(\omega^{\alpha}\right)_{\alpha=0,1}$ and $\left(\pi_{\dot{\alpha}}\right)_{\dot{\alpha}=\dot{0}, \dot{1}}$. First, we assumed that the momentum-space functions $f\left(\bar{\pi}_{\alpha}, \pi_{\dot{\alpha}}\right)$ can be expanded in terms of the power series terms $\left(\bar{\pi}_{0}\right)^{a}\left(\bar{\pi}_{1}\right)^{b}(a, b=0,1,2, \cdots)$. Then, we proposed the Fourier transform of $f\left(\bar{\pi}_{\alpha}, \pi_{\dot{\alpha}}\right), \mathcal{F}\left[f\left(\bar{\pi}_{\alpha}, \pi_{\dot{\alpha}}\right)\right]$, as a complex integral with respect to the variables $\bar{\pi}_{\alpha}$, which transforms $\left(\bar{\pi}_{0}\right)^{0}\left(\bar{\pi}_{1}\right)^{0}=1$ to the complex function of $\omega^{\alpha}$ representing the delta function of Sato's hyperfunction. By this definition, $\mathcal{F}\left[f\left(\bar{\pi}_{\alpha}, \pi_{\dot{\alpha}}\right)\right]$ is the twistor-space function $\tilde{f}\left(\omega^{\alpha}, \pi_{\dot{\alpha}}\right)$, which is expanded in terms of the power series terms $\left(\omega^{0}\right)^{-a-1}\left(\omega^{1}\right)^{-b-1}(a, b=0,1,2, \cdots)$. Next, we proposed the inverse Fourier transform of $\tilde{f}\left(\omega^{\alpha}, \pi_{\dot{\alpha}}\right), \mathcal{F}^{-1}\left[\tilde{f}\left(\omega^{\alpha}, \pi_{\dot{\alpha}}\right)\right]$, as a complex integral that transforms the complex function of $\omega^{\alpha}$ representing the delta function of Sato's hyperfunction to 1 . Using this definition, we showed that $\mathcal{F}^{-1}\left[\tilde{f}\left(\omega^{\alpha}, \pi_{\dot{\alpha}}\right)\right]$ is precisely the inverse map of $\mathcal{F}\left[f\left(\bar{\pi}_{\alpha}, \pi_{\dot{\alpha}}\right)\right]$, i.e., $\mathcal{F}^{-1} \mathcal{F}\left[f\left(\bar{\pi}_{\alpha}, \pi_{\dot{\alpha}}\right)\right]=f\left(\bar{\pi}_{\alpha}, \pi_{\dot{\alpha}}\right)$, $\mathcal{F F}^{-1}\left[\tilde{f}\left(\omega^{\alpha}, \pi_{\dot{\alpha}}\right)\right]=\tilde{f}\left(\omega^{\alpha}, \pi_{\dot{\alpha}}\right)$.

To the best of our knowledge, this study is the first to show that this Fourier transform is an isomorphism from $H^{0}\left(\mathbb{C P}^{1}, \mathcal{O}(n)\right)(n=0,1,2, \cdots)$ to $H^{1}\left(\mathbb{C P}^{1}, \mathcal{O}(-n-2)\right)$, and that the inverse Fourier transform is an isomorphism from $H^{1}\left(\mathbb{C P}^{1}, \mathcal{O}(-n-2)\right)$ to $H^{0}\left(\mathbb{C P}^{1}, \mathcal{O}(n)\right)$, from the perspective of Čech cohomology groups. In particular, owing to the Serre duality of the Čech cohomology groups, the mapping consisting of the inverse Fourier transform and the change of variables $\left(\bar{\pi}_{0}, \bar{\pi}_{0}\right) \mapsto\left(\omega^{0}, \omega^{1}\right)$ is an isomorphism from $H^{1}\left(\mathbb{C P}^{1}, \mathcal{O}(-n-2)\right)$ to its dual space $H^{0}\left(\mathbb{C P}^{1}, \mathcal{O}(n)\right)$.

Further, we demonstrated that the momentum-space functions to which the Fourier transform is applied are $\oplus_{n=0}^{\infty} H^{0}(L, \mathcal{O}(n+2+m)) \otimes H^{0}\left(\mathbb{C P}^{1}, \mathcal{O}(n)\right)(m \in$ $\mathbb{Z}$ ) and that the twistor-space functions to which the inverse Fourier transform is applied are $H^{1}(\mathbb{P} \mathbb{T}-L, \mathcal{O}(m))$.

We also showed that the representations of the operators $\hat{\bar{\pi}}_{\alpha}$ and $\hat{\omega}^{\alpha}$, which are well known in the twistor theory literature, are valid for this Fourier transform and its inverse. In addition, the application of these operators is closed on the 
momentum-space functions $\oplus_{m=-\infty}^{\infty} \oplus_{n=0}^{\infty} H^{0}(L, \mathcal{O}(n+2+m)) \otimes H^{0}\left(\mathbb{C P}^{1}, \mathcal{O}(n)\right)$ for the representation in which $\hat{\bar{\pi}}_{\alpha}$ reduces to $\bar{\pi}_{\alpha}$, and on the twistor-space functions $\oplus_{m=-\infty}^{\infty} H^{1}(\mathbb{P} \mathbb{T}-L, \mathcal{O}(m))$ for the representation in which $\hat{\omega}^{\alpha}$ reduces to $\omega^{\alpha}$.

In the real twistor space $\mathbb{R P}^{3}$, it is shown using Fourier transform that the threeparticle MHV and googly MHV super-amplitudes in $\mathcal{N}=4 \mathrm{SYM}$, which are the seed amplitudes for the twistor BCFW recursion, contain the so-called sign factor 617. This sign factor is the sign of the product of two twistors and one infinity twistor; therefore, it breaks the conformal invariance. Additionally, the analogous factor also appears in the amplitudes in $\mathcal{N}=8$ supergravity. The origin of the sign factor is considered to be a consequence of the fact that the amplitudes are functions on $\mathbb{R P}^{3}$. In the complex twistor space $\mathbb{C P}^{3}$, the amplitudes are elements of the Čech cohomology group, rather than functions 20. If the amplitudes are treated as elements of the Čech cohomology group on $\mathbb{C P}^{3}$, the sign factor will not appear, because it will be incorporated within the Čech cohomological structure [6]. Hence, in the future studies, we will reveal the origins of the sign factor by studying the manner in which the sign factor is incorporated within the Čech cohomological structure of the Fourier transform formulated in this study.

We treated the twistor-space functions as a Čech cohomology group. However, in another formulation, they can also be treated as a Dolbeault cohomology group 21. This formulation is in good agreement with twistor string theory. Therefore, in the future, we will consider a concrete integral formulation of the Fourier transform in terms of Dolbeault cohomology.

Finally, we note that the Fourier transform from momentum space to dual twistor space, i.e., the dual space of twistor space, can be defined in the same manner as in this paper.

\section{Acknowledgments}

I would like to thank S. Deguchi for his insightful comments.

\section{References}

[1] E. Witten, Perturbative gauge theory as a string theory in twistor space, Commun. Math. Phys. 252 (2004), 189-258; e-print arXiv:hep-th/0312171

[2] H. Elvang and Y. -t. Huang, Scattering amplitudes, ; e-print arXiv:1308.1697] [hepth].

H. Elvang and Y. -t. Huang, Scattering Amplitudes in Gauge Theory and Gravity (Cambridge University Press, Cambridge, 2015).

[3] I. Bena, Z. Bern, D. A. Kosower and R. Roiban, Loops in twistor space, Phys. Rev. D71 (2005), 106010; e-print arXiv:hep-th/0410054

[4] R. Britto, F. Cachazo and B. Feng, New recursion relations for tree amplitudes of gluons, Nucl. Phys. B715 (2005), 499-522; e-print arXiv:hep-th/0412308

[5] R. Britto, F. Cachazo, B. Feng and E. Witten, Direct Proof of the Tree-Level Scattering Amplitude Recursion Relation in Yang-Mills Theory, Phys. Rev. Lett. 94 (2005), 181602; e-print arXiv:hep-th/0501052. 
[6] L. Mason and D. Skinner, Scattering amplitudes and BCFW recursion in twistor space, JHEP 1001 (2010), 064; e-print arXiv:0903.2083 [hep-th].

[7] N. Arkani-Hamed, F. Cachazo, C. Cheung and J. Kaplan, The S-matrix in twistor space, JHEP 03 (2010), 110; e-print arXiv:0903.2110 [hep-th].

[8] N. Arkani-Hamed, F. Cachazo, C. Cheung and J. Kaplan, A duality for the S matrix, JHEP 1003 (2010), 020; e-print arXiv:0907.5418 [hep-th].

[9] L. Mason and D. Skinner, Dual superconformal invariance, momentum twistors and Grassmannians, JHEP 0911 (2009), 045; e-print arXiv:0909.0250 [hep-th].

[10] S. Deguchi, S. Negishi, S. Okano and T. Suzuki, Canonical formalism and quantization of a massless spinning bosonic particle in four dimensions, Int. J. Mod. Phys. A29 (2014), 1450044; e-print arXiv:1309.4169 [hep-th].

[11] R. Penrose, Twistor algebra, J. Math. Phys. 8 (1967), 345-366.

[12] R. Penrose, Twistor quantisation and curved space-time, Int. J. Theor. Phys. 1 (1968), 61-99.

[13] R. Penrose and M. A. H. MacCallum, Twistor theory: an approach to the quantisation of fields and space-time, Phys. Rep. 6 (1972), 241-316.

[14] S. Deguchi and J. Note, (Pre-)Hilbert spaces in twistor quantization, J. Math. Phys. 54 (2013), 072304; e-print arXiv:1210.0349 [hep-th].

[15] A. Kaneko, Introduction to Hyperfunctions, Mathematics and its applications, Japanese series translated by Y. Yamamoto, edited by F. M. Arscott (KTK Scientific Publishers, Kluwer Academic Publishers, Dordrecht, 1988).

[16] S. A. Huggett and K. P. Tod, An Introduction to Twistor Theory, Second Edition, London Mathematical Society, Student Texts 4 (Cambridge University Press, Cambridge, 1994).

[17] R. O. Wells, Jr., Differential Analysis on Complex Manifolds, 3rd ed., Graduate Texts in Mathematics 65 (Springer-Verlag, New York, 2008).

S. Kobayashi, Complex Geometry (Iwanami Shoten Co., Ltd., Tokyo, 2005) [in Japanese].

[18] M. G. Eastwood and L. Hughston, Massless fields based on a line: explosion and annihilation, Twistor Newsletter 8 (1979), 43-47.

[19] R. J. Baston and M. G. Eastwood, The Penrose Transform: Its Interaction with Representation Theory, Oxford Mathematical Monographs 499 (Clarendon Press, Oxford, 1989).

M. G. Eastwood and A. M. Pilato, On the density of twistor elementary states, Pacific J. Math. 151 (1991), 201-215.

[20] R. Penrose, Twistor functions and sheaf cohomology, Twistor Newsletter 2 (1976), 3-12.

[21] R. O. Wells, Jr., Complex manifolds and mathematical physics, Bull. Amer. Math. Soc. (new series) 1 (1979), 296-336.

N. M. J. Woodhouse, Twistor cohomology without sheaves, Twistor Newsletter 2 (1976), 13-14. 